\title{
Video-assisted thoracoscopic image-guided spine surgery: evolution of 19 years of experience, from endoscopy to fully integrated 3D navigation
}

\author{
George Hanna, MD, ${ }^{1}$ Terrence T. Kim, MD, ${ }^{2}$ Syed-Abdullah Uddin, BS, ${ }^{1,3}$ Lindsey Ross, MD, ${ }^{1}$ and \\ J. Patrick Johnson, MD1
}

Departments of ${ }^{1}$ Neurological Surgery and ${ }^{2}$ Orthopaedics, Cedars-Sinai Medical Center, Los Angeles; and ${ }^{3}$ Riverside School of Medicine, University of California, Riverside, California

\begin{abstract}
OBJECTIVE The purpose of this study was to describe the evolution of thoracoscopic spine surgery from basic endoscopic procedures using fluoroscopy and anatomical localization through developmental iterations to the current technology use in which endoscopy and image-guided surgery are merged with intraoperative CT scanning.

METHODS The authors provided detailed explanations of their thoracoscopic spine surgery techniques, beginning with their early-generation endoscopy with fluoroscopic localization, which was followed with point surface matching techniques and early image guidance. The authors supplanted this with the modern era of image guidance, thoracoscopic spine surgery, and seamless integration that has reached its current level of refinement.

RESULTS A retrospective review of single-institution thoracoscopic procedures performed by the senior author over the course of 19 years yielded a total of 160 patients, including 73 women and 87 men. The mean patient age was 55 years, and the range included patients 16-94 years of age. There were no patients with worsened neurological function. One hundred sixteen patients underwent surgery for thoracic disc herniation, 18 for underlying neoplasms with spinal cord compression, 14 for osteomyelitis and discitis, 12 for thoracic deformity with neurological changes, and 8 for traumatic etiologies.
\end{abstract}

CONCLUSIONS More than 19 years of experience has revealed the benefits of integrating thoracoscopic spine surgery with intraoperative CT scanning and image-guided surgery, including direct decompression without manipulation of neural elements, superior 3D spatial orientation, and localization of complex spinal anatomy. With the exponential growth of machine learning, robotics, artificial intelligence, and advances in imaging techniques and endoscopic imaging, there may be further refinements of this technique on the horizon.

https://thejns.org/doi/abs/10.3171/2020.10.FOCUS20792

KEYWORDS minimally invasive thoracoscopy; VATS; video-assisted thoracoscopic spine surgery; image-guided endoscopic spine surgery; endoscopic thoracic discectomy; stereotactic navigation; IGS

$\mathrm{M}$ ODERN-DAY image-guided surgery (IGS) in cranial and spinal neurosurgery with advanced computers and frame-based technology expanded in the 1990s, but one of the earliest documented cases in which combined fluoroscopic and television technology was used to perform a stereotactic cordotomy was noted in the 1960s. ${ }^{1}$ Widespread use in various subspecialties has been expanded to represent the standard of care in cranial neurosurgery ${ }^{2-6}$ and has become commonplace in spine surgery. A reference frame can be adapted to any part of the skull and spinal column to navigate surgical procedures.
A unique part of the spinal column that poses a challenge to surgical access, approach, and visualization is the anterior and lateral thoracic spine. One of the more technically challenging procedures in spine surgery is a thoracic disc herniation, with greater complexity added in deformity, neoplasms, and infection. The high risk of neurological decline from a posterior midline approach has encouraged the development of different safe approaches for spinal cord decompression without neural manipulation and maximal safe decompression. As early as 1969, Perot and Munro recognized the need for safer approaches to decompress the spinal cord, and they performed a

ABBREVIATIONS ICT = intraoperative CT; IGS = image-guided surgery; MIS = minimally invasive surgery; OPLL = ossification of the posterior longitudinal ligament; VATS = video-assisted thoracoscopic surgery. 
novel transthoracic approach with good neurological outcome.

The first reported usage of video-assisted thoracoscopic surgery (VATS) of the spine was in 1993 and was considered the prototype for minimally invasive surgery (MIS) of the spine. ${ }^{8-11}$ It was difficult to use for a relatively infrequent disease (i.e., thoracic disc herniation, thoracic osteomyelitis, or thoracic neoplasm), early instruments were limited, and 3D orientation was difficult to interpret on a $2 \mathrm{D}$ screen. ${ }^{12-18}$ To improve on this technology, we merged and incorporated both IGS and fluoroscopy to point register to the anterior thoracic spine and correlate with endoscopic imaging to allow for better accuracy and familiarity, and we published the results of our early experience. ${ }^{19-22}$ However, due to the difficult calibration of this point-match registration system on the anterior spine, it was difficult to achieve IGS registration with the spinal anatomy. Therefore, it failed to gain widespread acceptance and incorporation at different centers..$^{20,23,24}$

We entered the second generation of this combined IGS and thoracoscopic surgery with the advent of the intraoperative CT (iCT) scanner in the form of the O-arm (Medtronic, Inc.) in 2006. This technology and advanced registration system allowed for more versatility, accurate registration, and widespread incorporation because this technology was already being used with IGS for posterior spinal instrumentation, and we published these results and techniques in 2014. ${ }^{25}$ As with all technologies, we have made continuous improvements in our IGS systems with refinements of our techniques, which has led to a superior workflow and maximal patient benefits under the senior spine surgeon's guidance in 160 patients treated over a period of 19 years at a single institution. This manuscript describes the evolution of early-generation combined IGS and VATS technology, which was difficult to easily reproduce and was supplanted with automatically registered iCT-which has fully matured in our experience and has begun to gain acceptance and usage again. ${ }^{26}$

\section{Methods}

We previously introduced and meticulously elucidated our approach for image-guided thoracoscopic spine surgery in 2014. ${ }^{25}$ In our third generation, which has evolved, we highlight the changes in our approach below.

\section{Key Sequences for Incision, Port Placement, and Optimization of Trajectory}

The key sequences are as follows: 1) iCT image acquisition; 2) localization for the optimal trajectories for our port placement using IGS; and 3) thoracic endoscopy and performance of the decompressive spinal procedure. We currently use the StealthStation S8 navigation system (Medtronic, Inc.) for registration and guidance, which integrates with the O-arm iCT (Fig. 1).

\section{Position and Preparation}

Deflation of the ipsilateral lung allows for adequate visualization of the operative field. It is essential that the patient is in a true lateral position in order for visualization and localization to integrate seamlessly. The surgeon

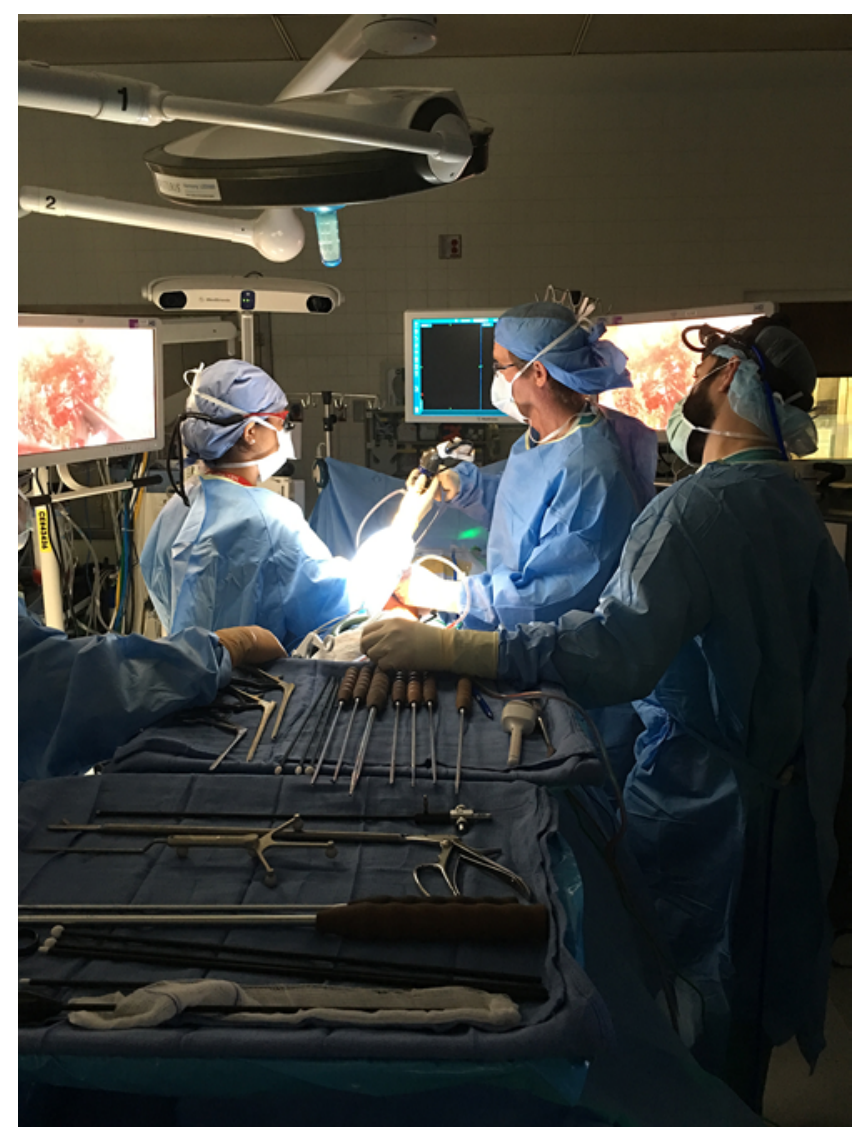

FIG. 1. Setup of operating room with the operating surgeon working with the anterior access ports. The monitor with the thoracoscopic images and the IGS monitor are both facing the surgeon. The IGS camera array is also facing the posteriorly placed reference frame.

operates from the ventral side of the patient as they maintain the trajectories of the working ports aimed posteriorly toward the disc space and central canal. A second assistant is on the dorsal side of the patient for facilitation of video camera positioning and extra instrument preparation.

\section{Radiographic Localization and Placement of Spinal Reference Frame}

The current localization is done entirely with iCT and the navigation system. After preparing and draping the patient, including the chest for thoracoscopy access, a separate dorsal midline incision is made at a similar thoracic operative level for spinous process reference frame attachment. Localization is confirmed in relation to the reference frame. Most thoracic disc herniations or pathologies have a distinctive radiographic appearance (e.g., calcified disc herniation, distinct vertebral osteophytes, etc.), which can be confirmed on a preoperative CT scan. We always recommend obtaining a preoperative scan for any patient with thoracic pathology who may undergo resection.

\section{Navigation Registration}

As compared to our last generation, we now use the StealthStation S8 (Medtronic, Inc.), with automatic reg- 


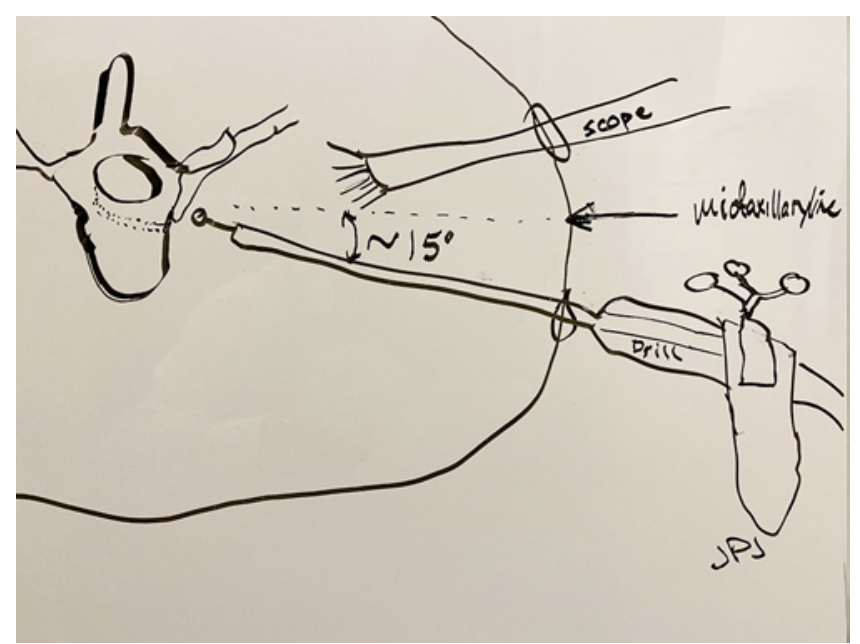

FIG. 2. Schematic drawing depicting the instruments in the ports, which include a drill and a scope, angled at $15^{\circ}$, in relation to the midaxillary line and in the trajectory of the rib head-pedicle junction at the level of the pathology.

istration of the CT images obtained by the $\mathrm{O}$-arm, and this process is carried out by the software installed in the StealthStation. We attach and calibrate a reference frame to our instruments, with the most important instrument being the drill. We typically use a Midas R or TU tool with a No. 33 bit (Medtronic, Inc.).

\section{Port Placement}

The 3 thoracoscopic port placements are placed in a triangular manner, with the first endoscope port placed in the posterior axillary line that is directly perpendicular to the level of the spinal pathology. The subsequent 2 working ports are placed in the anterior axillary line approximately 3-4 cm rostral and caudal to the operative level of interest. The port placement anterior and posterior to the midaxillary line results in approximately a $15^{\circ}$ angulation of the instruments in the working ports (Fig. 2). A trocar device can be used if needed to penetrate the tough muscular abdominal wall. Additionally, the exact sites of the port placement are now determined based on the most favorable trajectory as directed by navigation visualization before the incision is made (Fig. 3).

\section{Surgical Instruments}

We have previously described the instruments used, ${ }^{25}$ however, it is important to note that although the $0^{\circ}$ endoscopes are used for the majority of cases, occasionally the $15^{\circ}$ endoscopes are used when needed with difficult visualization. The high-speed drill is the most important instrument in a thoracoscopic procedure; therefore, we track the drill in real time with an attached reference frame.

\section{Image-Guided Thoracoscopic Procedure}

Image guidance is used to localize the level of pathology, which can include disc herniation, deformity, neoplasm, infection, or trauma. Once the level of interest is identified, the pleura is opened as previously described ${ }^{25}$ In order to expand the working corridor, we identify the rib head overlying the pedicle and/or disc space of interest. It can be drilled out so as to expand the bony foramen, as is the disc space posteriorly. This working corridor then allows the surgeon more space ventral to the spinal cord.

The image-guidance system then provides real-time orthogonal 3D views as the reference tracking device is attached and calibrated to any working instrument. The goal is to achieve adequate decompression circumferentially, and so the ultimate target in trajectory views is the contralateral pedicle (Fig. 3). A large working cavity in the posterior one-third of the vertebrae is created crossing the disc space and allows for safe access ventral to the central canal and spinal cord. The ability to visualize the deep bony anatomy allows the surgeon to avoid injury to surrounding neurovascular structures.

Once the dura mater is visualized from superior to inferior disc levels as well as from ipsilateral to contralateral pedicles, the adequacy of the decompression is confirmed. Additional localization and exploration can be assessed at the end of the procedure, and hemostasis is then achieved. If necessary, a repeat iCT scan can be performed to confirm the adequacy of the decompression.

\section{Results}

\section{Patient Demographics and Results}

Our combined multigenerational 19-year experience with video-assisted, image-guided MIS thoracoscopic procedures was successfully performed in 160 patients (73 women and 87 men). The mean age of the patients was 55 years (range 16-94 years). Patient characteristics and demographics are listed in Table 1.

The most common diagnosis was herniated disc causing myelopathy in patients with 1-, 2-, 3-, 4-, and 5-level disc herniations, and the remainder included patients with tumors, trauma, deformity, discitis/osteomyelitis, ossification of the posterior longitudinal ligament (OPLL), and ankylosing spondylitis. There was a total of 116 patients with symptomatic disc herniations, of which 98 were single-level, 8 were 2-level, 5 were 3 -level, 2 were 4 -level, and 3 were 5-level disc herniations. Fourteen patients underwent surgery for discitis/osteomyelitis, one of whom had Pott's disease. A total of 8 patients had a traumatic etiology that necessitated surgery. Twelve patients had degenerative kyphotic deformity, including Scheuermann's kyphosis, without underlying neoplasm, infection, or trauma. Eighteen patients were operated on for neoplastic etiologies that included metastatic renal cell carcinoma, breast carcinoma, nerve sheath tumors, spindle cell tumor, intraspinal hemorrhagic hemangioma, leiomyosarcoma, parotid carcinoma, plasmacytoma, and chronic myelogenous leukemia.

The mean blood loss was $476 \mathrm{ml}$ (range 20-7000 ml), and the mean operative time was 292 minutes (range 90-679 minutes). Chest tubes were placed to water seal at the end of the case and maintained for an average of 24 hours, although 6 patients required chest tube drainage for 48 hours or more due to symptomatic effusion or underlying respiratory disease. Patients were typically followed for 1 year or until all symptoms had resolved (range 6 months -10 years). 


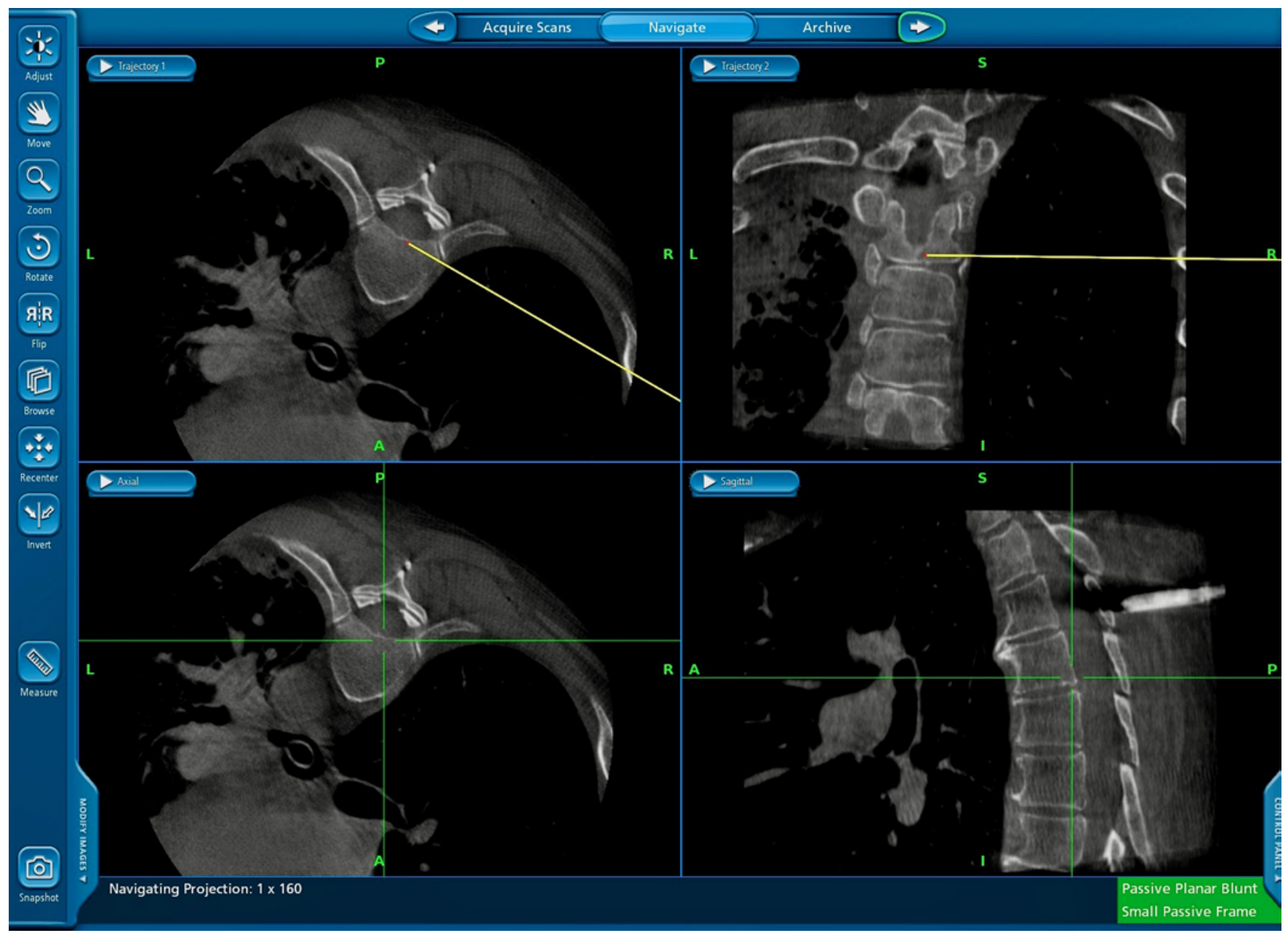

FIG. 3. Navigation screen on the Medtronic StealthStation S8 demonstrating a trajectory view in two planes and axial and sagittal views of the spine. The surgeon is using this view to determine the optimal location of port placement and to determine the optimal trajectory for the decompression. Please note the trajectory starting at the rib head and continuing close to the spinal canal en route to the contralateral pedicle.

The total number of discectomies performed for any etiology was a total of 143 disc levels (Table 2). The most common level operated on was T11-12 with 25 total discectomies, followed by T7-8 with 22 discectomies. There were no surgery-related deaths in our 19-year experience. There were no patients who had worsening neurological dysfunction and no reported significant lung, vascular (e.g., aorta or inferior vena cava), and sympathetic injuries. Although we had patients with CSF leakage associated with large, calcified disc herniations that were managed with lumbar drainage, only 1 patient required return to the operating room for repair. Additionally, 6 of our total 160 patients went back to the operating room for residual disc herniation or multilevel OPLL (Table 1).

\section{Discussion}

\section{History of Transthoracic Approaches for Thoracic Disc Surgery}

In 1969, Perot and Munro had summarized the high rate of neurological decline reported in the literature up to that point (i.e., 16/91 [17.5\%] of patients who had resultant paraplegia after midline laminectomies for decompression of thoracic disc herniations) and had presented a series of 2 patients in whom they performed a novel transthoracic approach with good neurological outcome.? Interestingly, in 15/16 of those patients who had paraplegia there was a central disc herniation and, although costotransversectomy and transpedicular approaches are feasible, we previously recommended transthoracic approaches for these central herniations and advised practitioners to use the prior approaches for paracentral herniations. ${ }^{20}$

\section{Early Localization in Thoracoscopic Spine Surgery}

Different treatments addressed for thoracoscopic spine pathologies include sympathectomy, ${ }^{27}$ discectomy, ${ }^{28}$ vertebral biopsy, anterior release, ${ }^{28}$ vertebral corpectomy, internal fixation, arthrodesis ${ }^{29}$ and tumor resection. ${ }^{26,30}$ Prior to the integration of image guidance, cumbersome techniques were used to localize the level of interest, including CT-guided methylene blue injection into the appropriate 
TABLE 1. Demographics and complications in patients who underwent thoracoscopic spine surgery

\begin{tabular}{lc}
\hline \multicolumn{1}{c}{ Variable } & Value \\
\hline No. of pts & 160 \\
\hline Sex (\%) & $87(54)$ \\
\hline Male & $73(46)$ \\
\hline Female & 55 \\
\hline Age in yrs & $16-94$ \\
\hline Mean & $476(20-7000)$ \\
\hline Range & $292(90-679)$ \\
\hline Mean estimated blood loss in ml (range) & $1(0.6)$ \\
\hline Mean op time in mins (range) & $6(3.8)$ \\
\hline Pts w/ complications requiring return to OR (\%) & \\
\hline CSF leak & \\
\hline Residual compressive disc or multifocal & $116(72.5)$ \\
\hline OPLL & $18(11.3)$ \\
\hline Underlying pathologies (\%) & $14(8.8)$ \\
\hline Thoracic disc herniation & $12(7.5)$ \\
\hline Neoplastic & $8(5)$ \\
\hline Discitis/osteomyelitis & \\
\hline Kyphotic deformity & \\
\hline Trauma & \\
\hline
\end{tabular}

$\mathrm{OR}=$ operating room; pts = patients.

level preoperatively. ${ }^{29}$ Although several variations of thoracotomy were used, only basic video endoscopy was being used during the surgical procedure when a 3D anatomical understanding was necessary, ${ }^{12,19}$ and so thoracoscopic procedures failed to be integrated in a widespread fashion.

\section{First-Generation Image Guidance and Thoracoscopic Disc Surgery}

First-generation image-guidance technology required that the surgeon either obtain a CT scan with the patient under general anesthesia ${ }^{31,32}$ or use historical CT data and do a manual point-to-point registration. ${ }^{13,19,20,33}$ These early registration protocols required matching 3 distinct radiographic points on the axial CT scans with 3 anatomical points on the spinal column. In the case of VATS, the major limitation was the inability to efficiently register the preoperative CT scan to the anterior spinal column because of the lack of distinct anatomical contours that could be accurately identified. These factors limited the clinical use of thoracoscopy and VATS for many procedures.

\section{Evolution of VATS With Modern-Era IGS Using ICT and Automatic Registration}

One of the positively disruptive changes in thoracoscopic spine surgery has been the advent of the mobile iCT scanners and automatic registration with the IGS workstation, which has streamlined the process of IGS thoracoscopic MIS of the spine. To date, we have had approximately 14 years of experience with this enhanced technology that continues to evolve and improve. The patient is positioned, and a reference clamp is attached to a spinous process in
TABLE 2. Levels of operation in our thoracoscopic disc surgery experience

\begin{tabular}{cc}
\hline Thoracoscopic Disc Levels & Total No. Treated \\
\hline T11-12 & 25 \\
\hline T10-11 & 15 \\
\hline T9-10 & 13 \\
\hline T8-9 & 14 \\
\hline T7-8 & 22 \\
\hline T6-7 & 19 \\
\hline T5-6 & 11 \\
\hline T4-5 & 7 \\
\hline T3-4 & 6 \\
\hline T2-3 & 11 \\
\hline
\end{tabular}

the vicinity of the level of pathology, followed by an iCT scan. Once the CT scanning process is completed, the images are directly loaded to a computer workstation, specifically the StealthStation (Medtronic, Inc.), where software renders a 3D reconstruction, automatically registers the images in relation to the reference frame and spine, and eliminates the logistics and potential error that previously affected the procedural accuracy of IGS. The efficiency and accuracy of this system has continued to improve with the current-generation StealthStation (S8; Medtronic, Inc.).

Previous localization has been largely eliminated using an integrated iCT and IGS system, whereas previous techniques relied on level counting from the pelvis, the skull base, or even required placement of a percutaneous pedicle marker in the fluoroscopy suite. ${ }^{34}$ This paper demonstrates our 19-year institutional experience with the various thoracic spinal pathologies that were addressed, including disc herniations (Fig. 4), tumors, infection, trauma, and deformity, which were preferentially treated using MIS procedures with IGS and VATS technologies.

The senior author has seen improved workflow, efficiency, and accuracy in these procedures as a result of experience, improved technology, and superior imaging quality. Although posterior lateral and oblique approaches exist, they offer inferior visualization and often lead to destabilization requiring arthrodesis and instrumentation. Studies have demonstrated that thoracoscopic discectomies do not necessarily disrupt the stability of the spinal column postprocedure, which is an advantageous feature over some posterior approaches that lead to spinal instability, less superior decompression, and necessity for instrumentation. ${ }^{35}$

The inherent limitation of this paper is that it reports only our current institutional experience-and with specific pathologies such as herniated discs, deformity, trauma, tumors, vertebral osteomyelitis, and discitis. Nevertheless, it demonstrates the experience of our senior author over the course of 2 decades of the evolution of a seamless workflow fully integrating the live visualization of VATS and $\mathrm{iCT}$ with superior image guidance. We previously demonstrated the advantages of thoracoscopic over open thoracotomy procedures. ${ }^{20}$ As new technology emerges with advanced imaging techniques, robotics, artificial intelligence, and machine learning, we will undoubtedly be 

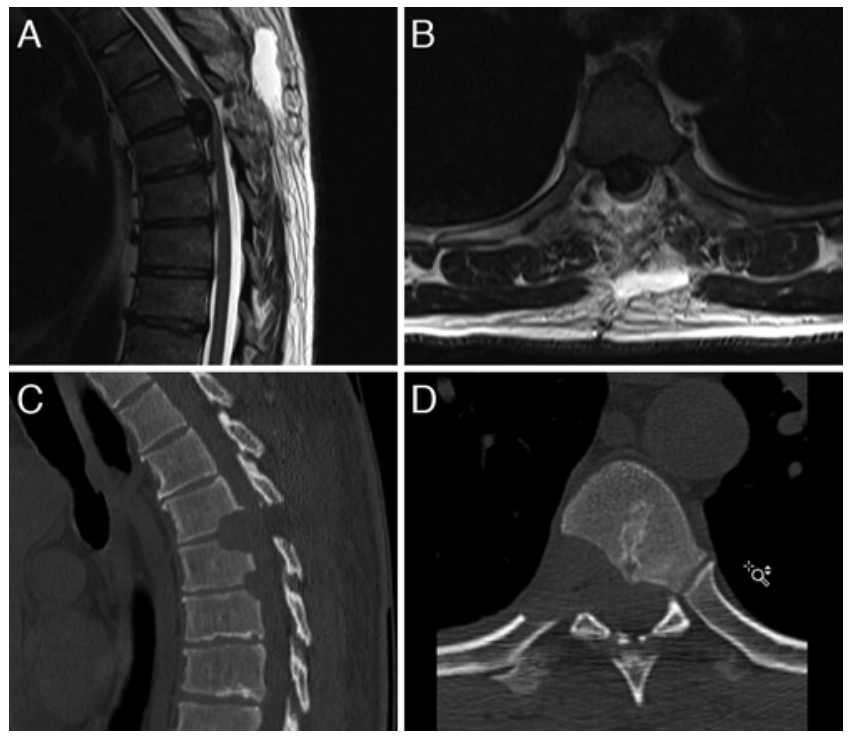

FIG. 4. A and B: Preoperative thoracic spine T2-weighted MRI, sagittal view (A) and axial view (B), demonstrating giant T5-6 central thoracic and smaller T6-7 disc herniations. The patient had prior laminectomies performed at $\mathrm{T} 6$ by a previous surgeon. $\mathrm{C}$ and $\mathrm{D}$ : Postoperative thoracic spine CT with sagittal view $(C)$ and axial view (D) demonstrating bony decompression at T5-6 and T6-7.

able to visualize and treat a wider range of thoracic spine diseases thoracoscopically. ${ }^{36}$ Other institutions have reported favorable outcomes and safety profile data for thoracoscopic discectomy. ${ }^{37}$ Other applications to the fields of deformity correction and anterior instrumentation have been reported and serve as another potential avenue for expansion of this technology. ${ }^{28,38-40}$ The safe approach to tumor resection with good visualization ${ }^{30,41}$ has led to the emergence and reapplication of this technology to a thoracic schwannoma at another center recently. ${ }^{26}$

\section{Conclusions}

VATS has arguably remained the only true MIS procedure for the thoracic spine with real-time visualization, iCT, superior integrative 3D technology, and a seamless workflow. The evolution of this procedure has demonstrated efficacy, superiority, and safety as compared to alternative approaches and open thoracotomies. More than 19 years of experience has revealed the benefits of this approach, including direct decompression in many different underlying pathologies without manipulation of neural elements, superior 3D spatial orientation, and localization of complex spinal anatomy. While these techniques have gained variable acceptance at other centers, this approach offers a powerful tool for a relatively rare but complex pathology. With the exponential growth of machine learning, robotics, artificial intelligence and advances in imaging techniques and endoscopic imaging, there may be further refinements of this technique on the horizon.

\section{Acknowledgments}

We acknowledge the Cedars-Sinai Medical Center anesthe- siologists Robert Naruse, MD, and Omar Durra, MD, as well as the operating room and IGS technicians Michael Arango, Mario Lujan, and Randy De Sesto for their expertise in facilitating these complex procedures and contributions to the information on technology. We also acknowledge Patricia Vetter for her artistic contributions.

\section{References}

1. Fox JL, Green RC. Stereotaxic surgery using a television guidance system. II. Percutaneous cordotomy. Acta Neurochir (Wien). 1969;21(1):31-42.

2. Silva D, Belsuzarri T, Barnett GH. Image-guided surgery for meningioma. Handb Clin Neurol. 2020;170:201-207.

3. Schmale IL, Vandelaar LJ, Luong AU, et al. Imageguided surgery and intraoperative imaging in rhinology: clinical update and current state of the art. Ear Nose Throat J. Published online May 26, 2020. doi: $10.1177 / 0145561320928202$

4. Barrett TF, Dyvorne HA, Padormo F, et al. First application of 7-T magnetic resonance imaging in endoscopic endonasal surgery of skull base tumors. World Neurosurg. 2017;103: 600-610.

5. Parikh NR, Kundu P, Levin-Epstein R, et al. Time-driven activity-based costing comparison of stereotactic radiosurgery to multiple brain lesions using single-isocenter versus multiple-isocenter technique. Int J Radiat Oncol Biol Phys. 2020;108(4):999-1007.

6. Giaj-Levra N, Borghetti P, Bruni A, et al. Current radiotherapy techniques in NSCLC: challenges and potential solutions. Expert Rev Anticancer Ther. 2020;20(5):387-402.

7. Perot PL Jr, Munro DD. Transthoracic removal of midline thoracic disc protrusions causing spinal cord compression. $J$ Neurosurg. 1969;31(4):452-458.

8. Huang TJ, Hsu RW, Sum CW, Liu HP. Complications in thoracoscopic spinal surgery: a study of 90 consecutive patients. Surg Endosc. 1999;13(4):346-350.

9. Landreneau RJ, Hazelrigg SR, Mack MJ, et al. Postoperative pain-related morbidity: video-assisted thoracic surgery versus thoracotomy. Ann Thorac Surg. 1993;56(6):1285-1289.

10. Mack MJ, Regan JJ, Bobechko WP, Acuff TE. Application of thoracoscopy for diseases of the spine. Ann Thorac Surg. 1993;56(3):736-738.

11. Rosenthal D, Rosenthal R, de Simone A. Removal of a protruded thoracic disc using microsurgical endoscopy. A new technique. Spine (Phila Pa 1976). 1994;19(9):1087-1091.

12. Anand N, Regan JJ. Video-assisted thoracoscopic surgery for thoracic disc disease: Classification and outcome study of 100 consecutive cases with a 2-year minimum follow-up period. Spine (Phila Pa 1976). 2002;27(8):871-879.

13. Bolger $C$, Wigfield $C$. Image-guided surgery: applications to the cervical and thoracic spine and a review of the first 120 procedures. J Neurosurg. 2000;92(2)(suppl):175-180.

14. Holly LT, Bloch O, Johnson JP. Evaluation of registration techniques for spinal image guidance. J Neurosurg Spine. 2006;4(4):323-328.

15. Kalfas IH, Kormos DW, Murphy MA, et al. Application of frameless stereotaxy to pedicle screw fixation of the spine. $J$ Neurosurg. 1995;83(4):641-647.

16. Kim KD, Johnson JP, Babbitz JD. Image-guided thoracic pedicle screw placement: a technical study in cadavers and preliminary clinical experience. Neurosurg Focus. 2001; 10(2):E2.

17. Kim KD, Johnson JP, Masciopinto JE, et al. Universal calibration of surgical instruments for spinal stereotaxy. Neurosurgery. 1999;44(1):173-178.

18. Kim KD, Johnson JP, Bloch BS O, Masciopinto JE. Computer-assisted thoracic pedicle screw placement: an in vitro feasibility study. Spine (Phila Pa 1976). 2001;26(4):360-364. 
19. Johnson JP, Stokes JK, Oskouian RJ, et al. Image-guided thoracoscopic spinal surgery: a merging of 2 technologies. Spine (Phila Pa 1976). 2005;30(19):E572-E578.

20. Johnson JP, Filler AG, McBride DQ. Endoscopic thoracic discectomy. Neurosurg Focus. 2000;9(4):e11.

21. Oskouian RJ Jr, Johnson JP, Regan JJ. Thoracoscopic microdiscectomy. Neurosurgery. 2002;50(1):103-109.

22. Oskouian RJ, Johnson JP. Endoscopic thoracic microdiscectomy. J Neurosurg Spine. 2005;3(6):459-464.

23. Haberland N, Ebmeier K, Grunewald JP, et al. Incorporation of intraoperative computerized tomography in a newly developed spinal navigation technique. Comput Aided Surg. 2000; 5(1):18-27.

24. Holly LT, Bloch O, Obasi C, Johnson JP. Frameless stereotaxy for anterior spinal procedures. J Neurosurg. 2001;95(2) (suppl):196-201.

25. Johnson JP, Drazin D, King WA, Kim TT. Image-guided navigation and video-assisted thoracoscopic spine surgery: the second generation. Neurosurg Focus. 2014;36(3):E8.

26. Sweeney J, Zyck S, Crye M, Galgano M. Novel single-staged posterior retropleural approach with thoracoscopic guidance for resection of a thoracic dumbbell schwannoma. Cureus. 2020;12(1):e6548.

27. Chen S, Zhang P, Chai T, et al. T3 versus T4 video-assisted thoracoscopic sympathectomy for palmar hyperhidrosis: a protocol for a systematic review and meta-analysis. Medicine (Baltimore). 2019;98(42):e17272.

28. Arlet V. Anterior thoracoscopic spine release in deformity surgery: a meta-analysis and review. Eur Spine J. 2000; 9(suppl 1):S17-S23.

29. Boriani S, Biagini R, De Iure F, et al. Two-level thoracic disc herniation. Spine (Phila Pa 1976). 1994;19(21):2461-2466.

30. Ghostine S, Vaynman S, Schoeb JS, et al. Image-guided thoracoscopic resection of thoracic dumbbell nerve sheath tumors. Neurosurgery. 2012;70(2):461-468.

31. Assaker R, Reyns N, Pertruzon B, Lejeune JP. Image-guided endoscopic spine surgery: Part II: clinical applications. Spine (Phila Pa 1976). 2001;26(15):1711-1718.

32. Assaker R, Cinquin P, Cotten A, Lejeune JP. Image-guided endoscopic spine surgery: Part I. A feasibility study. Spine (Phila Pa 1976). 2001;26(15):1705-1710.

33. Faciszewski T, Winter RB, Lonstein JE, et al. The surgical and medical perioperative complications of anterior spinal fusion surgery in the thoracic and lumbar spine in adults. A review of 1223 procedures. Spine (Phila Pa 1976). 1995; 20(14):1592-1599.

34. Binning MJ, Schmidt MH. Percutaneous placement of radiopaque markers at the pedicle of interest for preoperative localization of thoracic spine level. Spine (Phila Pa 1976). 2010;35(19):1821-1825.
35. Broc GG, Crawford NR, Sonntag VK, Dickman CA. Biomechanical effects of transthoracic microdiscectomy. Spine (Phila Pa 1976). 1997;22(6):605-612.

36. Harada GK, Siyaji ZK, Younis S, et al. Imaging in spine surgery: current concepts and future directions. Spine Surg Relat Res. 2019;4(2):99-110.

37. Wait SD, Fox DJ Jr, Kenny KJ, Dickman CA. Thoracoscopic resection of symptomatic herniated thoracic discs: clinical results in 121 patients. Spine (Phila Pa 1976). 2012;37(1): $35-40$.

38. Newton PO, Upasani VV, Lhamby J, et al. Surgical treatment of main thoracic scoliosis with thoracoscopic anterior instrumentation. Surgical technique. J Bone Joint Surg Am. 2009; 91(suppl 2):233-248.

39. Newton PO, White KK, Faro F, Gaynor T. The success of thoracoscopic anterior fusion in a consecutive series of 112 pediatric spinal deformity cases. Spine (Phila Pa 1976). 2005;30(4):392-398.

40. Horn EM, Henn JS, Lemole GM Jr, et al. Thoracoscopic placement of dual-rod instrumentation in thoracic spinal trauma. Neurosurgery. 2004;54(5):1150-1154.

41. Campos WK, Gasbarrini A, Boriani S. Case report: Curetting osteoid osteoma of the spine using combined videoassisted thoracoscopic surgery and navigation. Clin Orthop Relat Res. 2013;471(2):680-685.

\section{Disclosures}

Dr. Kim has a Medtronic research grant and is a consultant for Medtronic and DePuy Synthes.

\section{Author Contributions}

Conception and design: Johnson, Hanna. Acquisition of data: Johnson, Hanna, Ross. Analysis and interpretation of data: Johnson, Hanna, Kim. Drafting the article: Johnson, Hanna. Critically revising the article: all authors. Reviewed submitted version of manuscript: all authors. Approved the final version of the manuscript on behalf of all authors: Johnson. Statistical analysis: Johnson, Hanna, Uddin. Administrative/technical/ material support: Hanna, Uddin. Study supervision: Johnson.

\section{Correspondence}

J. Patrick Johnson: Cedars-Sinai Medical Center, Los Angeles, CA. patrick.johnson@cshs.org. 